\title{
COVID-19 uncertainty and sleep: the roles of perceived stress and intolerance of uncertainty during the early stage of the COVID-19 outbreak
}

\author{
Dan $\mathrm{Wu}^{1}$, Tingzhong Yang ${ }^{2}$, Daniel L. Hall ${ }^{3}$, Guihua Jiao ${ }^{4}$, Lixin Huang ${ }^{1}$ and Can Jiao ${ }^{1 *}$
}

\begin{abstract}
Background: The COVID-19 pandemic brings unprecedented uncertainty and stress. This study aimed to characterize general sleep status among Chinese residents during the early stage of the outbreak and to explore the network relationship among COVID-19 uncertainty, intolerance of uncertainty, perceived stress, and sleep status.

Methods: A cross-sectional correlational survey was conducted online. A total of 2534 Chinese residents were surveyed from 30 provinces, municipalities, autonomous regions of China and regions abroad during the period from February 7 to 14, 2020, the third week of lockdown. Final valid data from 2215 participants were analyzed. Self-report measures assessed uncertainty about COVID-19, intolerance of uncertainty, perceived stress, and general sleep status. Serial mediation analysis using the bootstrapping method and path analysis were applied to test the mediation role of intolerance of uncertainty and perceived stress in the relationship between uncertainty about COVID-19 and sleep status.
\end{abstract}

Results: The total score of sleep status was $4.82(S D=2.72)$. Age, place of residence, ethnicity, marital status, infection, and quarantine status were all significantly associated with general sleep status. Approximately half of participants (47.1\%) reported going to bed after 12:00 am, 23.0\% took 30 min or longer to fall asleep, and 30.3\% slept a total of $7 \mathrm{~h}$ or less. Higher uncertainty about COVID-19 was significantly positively correlated with higher intolerance of uncertainty $(r=0.506, p<0.001)$. The mediation analysis found a mediating role of perceived stress in the relationship between COVID-19 uncertainty and general sleep status $(\beta=0.015,95 \% C . I .=0.009-0.021)$. However, IU was not a significant mediator of the relationship between COVID-19 uncertainty and sleep $(\beta=0.009$, 95\%C.I. $=$ - 0.002-0.020). Moreover, results from the path analysis further showed uncertainty about COVID-19 had a weak direct effect on poor sleep ( $\beta=0.043, p<0.05$ ); however, there was a robust indirect effect on poor sleep through intolerance of uncertainty and perceived stress.

Conclusions: These findings suggest that intolerance of uncertainty and perceived stress are critical factors in the relationship between COVID-19 uncertainty and sleep outcomes. Results are discussed in the context of the COVID19 pandemic, and practical policy implications are also provided.

Keywords: Uncertainty about COVID-19, Intolerance of uncertainty, Perceived stress, Poor sleep

\footnotetext{
* Correspondence: jiaocan@szu.edu.cn

'School of Psychology, Shenzhen University, Shenzhen 518060, Guangdong, China

Full list of author information is available at the end of the article
}

(c) The Author(s). 2021 Open Access This article is licensed under a Creative Commons Attribution 4.0 International License, which permits use, sharing, adaptation, distribution and reproduction in any medium or format, as long as you give appropriate credit to the original author(s) and the source, provide a link to the Creative Commons licence, and indicate if changes were made. The images or other third party material in this article are included in the article's Creative Commons licence, unless indicated otherwise in a credit line to the material. If material is not included in the article's Creative Commons licence and your intended use is not permitted by statutory regulation or exceeds the permitted use, you will need to obtain permission directly from the copyright holder. To view a copy of this licence, visit http://creativecommons.org/licenses/by/4.0/. The Creative Commons Public Domain Dedication waiver (http://creativecommons.org/publicdomain/zero/1.0/) applies to the data made available in this article, unless otherwise stated in a credit line to the data. 


\section{Introduction}

The World Health Organization's (WHO's) China Country Office was informed of cases of pneumonia of unknown cause detected in Wuhan City, Hubei Province, on December 31, 2019 [1]. On January 23, in response to the growing COVID-19 epidemic, the Chinese government locked down Wuhan City [2]. During the first 14 days of lockdown, confirmed cases in China increased dramatically from 571 on January 23 to 28,060 on February 6 [1]. Due to the enforcement of the lockdown, most people were restricted to their homes. This confinement has resulted in a changed lifestyle, disrupted chronobiological rhythms, and impacted on mental health, including sleep issues [3-5]. An online survey demonstrated that $30 \%$ of participants among Wuhan residents had a sleep disorder during the incipient pandemic [6]. Similarly, an Italian study reported $42.2 \%$ of the sample had sleep disturbances and, among them, $17.4 \%$ reported moderate/severe insomnia in Italy, which was the first European country to enter a nationwide lockdown in 2020 [4]. A systematic review and metaanalysis demonstrated a global pooled prevalence rate of sleep problems among all populations was 35.7\% (95\% C.I.:29.4-42.4\%) which suggested the sleep problem was a major public health issue during the COVID-19 pandemic [5]. Sleep is an integral part of proper human function [7]. When facing COVID-19, sleep becomes essential because of its benefits for mental and physical health [5]. Poorer sleep quality is associated with increased susceptibility to viral infections, lower cognitive functioning, poorer job performance, and worsened mental health [8]. Understanding the influencing mechanism of sleep during the COVID-19 pandemic may help the residents to maintain good sleep status and provide support for the design and implementation of interventions for sleep health in pandemic conditions.

When confronted with such a sudden and actual disaster, people realize that uncertainty surrounds every aspect of life [9]. Uncertainty is one of the major cognitive and psychological stressors [10]. Further, factors relating to stress are one of the most important concomitants of sleep complaints in the general population [3, 11]. The concept of uncertainty in illness was defined as "the inability to determine the meaning of illness-related events" [12]. Applicable to COVID-19, uncertainty according to Mishel's initial definition is a cognitive state that occurs when the decision maker is unable to assign definite values to objects and events and/or is unable to accurately predict outcomes because of a lack of sufficient cues [12]. Since this unexpected COVID-19 pandemic has swept across the world, limited knowledge of COVID-19 for diagnosis and treatment, unpredictability of the natural course of contagious illness, and gross disruption of societal functioning and people's routines may all represent potential sources of uncertainty. Uncertainty has been identified as the greatest single psychological stressor for patients with a life-threatening illness [13]. Given the sustained uncertainties and challenges in managing COVID-19, it is likely that, if unmanaged, COVID-19 related uncertainty may persist and continue to impact sleep outcomes.

Intolerance of uncertainty (IU) relates to the cognitive unacceptability of uncertainty and represents a dispositional characteristic of those people who are more likely to "find ambiguity stressful and upsetting, believe uncertainty is negative and should be avoided, and have difficulty functioning in uncertain situations" [14, 15]. IU was first defined by Freeston and colleagues as "a relatively broad construct representing cognitive, emotional, and behavioral reactions to uncertainty in everyday life situations" [16]. Carleton further explicitly delineated the definition of IU that includes the triggering stimuli, the response, and the incapacity to endure the associated perception of uncertainty $[17,18]$. Although uncertainty is pervasive and inherent in people's daily lives, individuals who have a high degree of perceived uncertainty or who are high in IU experience might significantly impair their daily functioning [18-20]. A substantial body of research in the context of both normal time and COVID19 pandemic suggests that individual differences in IU foster stress, depression, and anxiety [19-22]. Several studies have uncovered the mutual relationship between IU and fear of COVID-19 [21, 23]. Disease fear increased IU, and higher IU aggravated fear of COVID-19, subsequently weakening people's well-being [23]. Theoretical conceptualizations posit that those high in IU are likely to rely on maladaptive behaviors as a coping mechanism when faced with uncertain and potentially aversive situations [24].

A meta-analysis shows that the association between IU and symptoms was observed across all kinds of mental disorders [25]. A significant association between IU and sleep disturbances in young adults and more specifically Iranian adults was examined [26]. A large body of evidence from clinical research has demonstrated that generalized anxiety disorder patients with greater IU suffer from sleep dysfunctions including longer sleep latency, decreased sleep duration, decreased total sleep efficiency, and increased waking periods during sleep time [27, 28]. Research by our group has also demonstrated strong associations between cancer-related uncertainty and insomnia severity among cancer survivors [29]. There is a plethora of studies on uncertainty for clinical patients with chronic diseases. However, research on uncertainty in a lethal and urgent infectious disease outbreak and its side effects is limited among non-patients $[25,30]$. The role of IU in sleep problems has not been studied extensively in the context of the COVID-19 pandemic $[5,15]$. 
Unknown is how IU impacts the public in cognitively processing a COVID-19-related stressor, as well as how their sleeping behaviors are influenced by such serious outbreak-related events.

Based on previous literature, IU appears to be an important element of cognitive vulnerability affecting sleep quality [28]. The current study seeks to explore the potential mechanism for influencing sleep status during the early stage of COVID-19 outbreak, and to examine how IU and perceived stress impact the relations between uncertainty about COVID-19 and sleep in a sample of Chinese general residents. The following four hypotheses and research questions were evaluated in this study. First, we expected a positive association between perceived uncertainty about COVID-19 and IU and, second, following prior studies, an association between IU and perceived stress $[19,31]$. Third, in line with previous research [32, 33], we predicted a positive association between stress and poor sleep. Finally, we treat as exploratory the possibility that IU and stress would play a mediating role in the relationship between uncertainty about COVID-19 and poor sleep.

\section{Methods}

\section{Study design and participants}

A cross-sectional correlational design and a combination of convenience and snowball sampling were utilized in this study. The online survey was conducted from February 7 to 14, 2020 (the third week after the lockdown of Wuhan City) based on the Wenjuanxing Platform (https://www.wjx.cn/app/survey.aspx) Twenty psychology students were trained as research assistants to recruit participants through WeChat and other major Chinese social networking platforms. Our sample covered 30 provinces, municipalities, autonomous regions of China, and regions abroad. A total of 2654 potential participants were contacted online, and 2534 agreed to participate in the survey. A more detailed description of the survey and the data can be found in $\mathrm{Wu}$ et al. [34]. Participants took approximately $15-\mathrm{min}$ to complete the questionnaire. The study protocol was approved by the Ethics Committee of Shenzhen University, and written consent was obtained from all participants prior to administration of the questionnaire.

\section{Measures}

The survey questionnaire covered five categories: (a) demographics, (b) sleep status, (c) uncertainty about COVID-19, (d) intolerance of uncertainty, and (e) perceived stress.

\section{Dependent variables \\ General sleep status}

General sleep status was measured by the 6-item Sleep Questionnaire regarding the Pittsburgh Sleep Quality Index (PSQI) [35]. The participants were asked about their sleep status during the past month by the following questions 1) how would you rate your sleep quality overall; 2) when have you usually gone to bed at night; 3 ) how long (in minutes) has it usually take to fall asleep; 4) how many hours of actual sleep did you get at night; 5) how often have you taken medicine to help you sleep; 6) how often have you had trouble staying awake [24]. Subjective sleep quality, sleep bedtime, sleep latency, sleep duration, use of sleeping medication, and daytime dysfunction were assessed. Each item is divided into four levels with a 4-point Likert scale. For subjective sleep quality, the responses were coded as $0=$ very good, $1=$ good, $2=$ bad, and $3=$ very bad. The answers for sleep bedtime were coded as $0=$ before $11: 00 \mathrm{pm}, 1=11: 00$ pm-12:00 am, $2=12: 00 \mathrm{am}-1: 00 \mathrm{am}$, and $3=$ after 1:00 $\mathrm{am}$. The options for sleep latency were scored as $0=$ less than $15 \mathrm{~min}, 1=16-30 \mathrm{~min}, 2=31-60 \mathrm{~min}$, and $3=$ more than $60 \mathrm{~min}$. The answers for sleep duration were scored as $0=$ more than $7 \mathrm{~h}, 1=6-7 \mathrm{~h}, 2=5-6 \mathrm{~h}$, and $3=$ less than $5 \mathrm{~h}$. The responses for use of sleeping medication and daytime dysfunction were all assigned as $0=$ none, $1=$ less than once a week, $2=$ once or twice a week, and $3=$ three or more times a week. When we factor-analyzed the sleep questionnaire in our study, we divided the components into two factors, which accounted for $52.75 \%$ of the variance. The components of sleep duration and use of sleep medication were more closely associated, and the other components were more closely associated in our subjects. The six-component scores were rated on a $0-3$ scale and then summed to yield a global sleep score which has a range of $0-18$, with higher scores reflecting poorer sleep quality. The total score of sleep status in this study has been found to have a good correlation $(r=0.699, p<0.01)$ with the single item of subjective sleep quality of the Pittsburgh Sleep Quality Index. The Cronbach's alpha coefficient of this sleep questionnaire was 0.713 , suggesting that its internal consistency was acceptable.

\section{Independent variables}

\section{Demographic characteristics}

The following sociodemographic information was collected during the survey: date of birth, gender, place of residence, ethnicity, marital status, educational attainment, occupation, and per capita annual family income. Specific experience with COVID-19, including infection and quarantine status, were also measured [34]. 


\section{Uncertainty about COVID-19}

The 10-item Uncertainty about COVID-19 Scale was developed with reference to the Mishel Uncertainty in Illness Scale [12]. We applied a 5-point Likert-type scale ranging from strongly agree to strongly disagree in tapping public perception of uncertainty about the COVID19 outbreak [34]. Item scores were summated to obtain a total uncertainty score. The higher the score, the greater the perceived uncertainty about the disease. The Kaiser-Meyer-Olkin measure of sampling adequacy was 0.866, and Bartlett's test of sphericity was significant $(P<0.001)$, suggesting the sample was factorable. The factor loading on each item exceeded 0.5. Finally, two distinct factors of "lack of information and clarity" and "unpredictability" were extracted, which accounted for $59 \%$ of the variance. The Cronbach's alphas coefficient was 0.75 for lack of information and clarity, and 0.85 for unpredictably, respectively. The reliability coefficient for the Uncertainty about COVID-19 Scale was 0.85, suggesting good reliability.

\section{Intolerance of uncertainty}

Intolerance of Uncertainty was measured by the 12-item Intolerance of Uncertainty Scale (IUS-12) [36]. The IUS12 was a widely used and reliable psychometric instrument that consisted of a stable two-factor structure, representing anxious and avoidance components of intolerance of uncertainty [36, 37]. Items are scored on a Likert scale ranging from 1 (not at all characteristic of me) to 5 (entirely characteristic of me), yielding possible total scores from 12 to 60 . Higher overall scores correspond to higher intolerance of uncertainty. The Cronbach's $\alpha$ coefficient for the Intolerance of Uncertainty Scale in this sample was 0.89 .

\section{Perceived stress}

Stress was measured by the Perceived Stress Scale, Chinese version (CPSS) which has good reliability and validity and has been widely used to assess perceived stress in community settings across China [38, 39]. This scale comprised 14 items that assessed the perception of stress during the month prior to the survey. Items were rated on a 5-point Likert-type scale and ranged from 1 (never) to 5 (very often). Item scores were summed to yield a total stress score. The higher the total score, the greater the perceived level of stress [38, 39]. The internal reliability of the CPSS in this sample, measured by Cronbach's $\alpha$, was 0.77 .

\section{Data analysis}

All survey data were entered into a Microsoft Excel database, and then imported into SPSS (version 22.0) and Amos 21.0 for statistical analysis. Descriptive statistics and univariate analysis on sleep status were conducted.
Cronbach's alpha coefficient and exploratory factor analysis were used to examine the reliability and validity of the sleep questionnaire, Uncertainty about COVID-19 Scale, Perceived Stress Scale, and Intolerance of Uncertainty Scale. Pearson correlational analysis was applied to explore the relationships among intolerance of uncertainty, uncertainty about COVID-19, perceived stress, and sleep status. Mediation analysis using the bootstrapping method was performed with PROCESS. Regards to the mediation model, the dependent variable was general sleep status, and uncertainty about COVID-19 was the independent variable. The mediators were IU and perceived stress, and the covariates were the significant demographic characteristics, infection, and quarantine status via univariate analysis. In the present study, the 95\% CI of the total effect, direct effect, and indirect effects was obtained with 5000 bootstrap resamples. A significant indirect effect via mediators between dependent and independent variables was identified if the $95 \% \mathrm{CI}$ does not contain zero [21]. Hypotheses were also tested by Structural Equation Modeling (SEM) with maximum likelihood estimation. SEM is a multivariate statistical analysis technique that is used to analyze structural relationships, including causal modeling, simultaneous equation modeling, path analysis, and so on [40]. This study used the path analysis to examine the network relationship among uncertainty about COVID-19, intolerance of uncertainty, perceived stress, and poor sleep. Finally, the model modification was also conducted to adjust the estimated model by adding or removing the path parameters [41]. Five of the most popular fit indices were selected to evaluate the overall fit of the model $[40,41]$. These fit indices were a Chi-square to degrees of freedom ratio $(x 2 / d f)$, the goodness-of-fit index (GFI), the comparative fit index (CFI), the root mean square error of approximation (RMSEA), standardized root mean square residual (SRMR) [41].

\section{Results}

The response rate could not be calculated because the study population was a convenience sample. A total of 2534 participants were surveyed, of whom 2215 (87.4\%) completed valid questionnaires. Among this general public sample, 54 respondents reported that they were infected by COIVD-19 and the remaining 2161 participants stated that they were uninfected. Over two-thirds of the participants were female $(67.2 \%)$ and urban residents $(68.7 \%), 75.3 \%$ were single $(75.3 \%)$, and $50.2 \%$ were aged 20 to 24 years. The characteristics of the respondents are summarized in Table 1 . The statistically significant differences in sociodemographic characteristics for total sleep scores were for age, place of residence, ethnicity, and marital status. Individual infection status and their friends, relatives, colleagues, and neighbors' 
Table 1 Demographic characteristics and total score of general sleep status

\begin{tabular}{|c|c|c|c|c|c|}
\hline Variables & $\mathrm{N}$ & $\%$ & Mean (SD) & $F / t$ & $p$ \\
\hline Age & & & & 6.800 & $<0.001^{* *}$ \\
\hline$<20$ & 411 & 18.6 & $4.49(2.64)$ & & \\
\hline $20-24$ & 1113 & 50.2 & $5.00(2.77)$ & & \\
\hline $25-29$ & 240 & 10.8 & $5.28(2.74)$ & & \\
\hline $30-39$ & 209 & 9.4 & $4.55(2.66)$ & & \\
\hline $40+$ & 242 & 10.9 & $4.36(2.53)$ & & \\
\hline Gender & & & & 0.235 & 0.628 \\
\hline Male & 726 & 32.8 & $4.86(2.80)$ & & \\
\hline Female & 1489 & 67.2 & $4.80(2.68)$ & & \\
\hline Place of residence & & & & 12.910 & $<0.001^{* *}$ \\
\hline Urban & 1522 & 68.7 & $4.96(2.74)$ & & \\
\hline Rural & 683 & 31.3 & $4.52(2.64)$ & & \\
\hline Ethnicity & & & & 8.269 & $0.004^{* *}$ \\
\hline Han & 2155 & 97.3 & $4.79(2.71)$ & & \\
\hline Minority & 60 & 2.7 & $5.82(2.87)$ & & \\
\hline Marital status & & & & 4.253 & $0.014^{*}$ \\
\hline Unmarried & 1699 & 75.3 & $4.88(2.73)$ & & \\
\hline Married & 517 & 23.3 & $4.57(2.67)$ & & \\
\hline Divorced/widowed & 29 & 1.3 & $5.72(2.52)$ & & \\
\hline Education & & & & 2.075 & 0.101 \\
\hline Junior high school or less & 196 & 8.8 & $4.68(2.82)$ & & \\
\hline High school & 233 & 10.5 & $5.04(2.86)$ & & \\
\hline Junior college & 263 & 11.9 & $5.13(3.02)$ & & \\
\hline College or higher & 1523 & 68.8 & $4.76(2.63)$ & & \\
\hline Occupation & & & & 0.621 & 0.648 \\
\hline Public official /professionals & 257 & 11.6 & $4.71(2.69)$ & & \\
\hline Enterprise personnel & 238 & 10.7 & $4.91(2.90)$ & & \\
\hline Commerce/service/operations & 215 & 9.7 & $5.03(2.61)$ & & \\
\hline Students & 1311 & 59.2 & $4.78(2.70)$ & & \\
\hline Others & 194 & 8.8 & $4.91(2.80)$ & & \\
\hline Household annual income (RMB) & & & & 0.750 & 0.522 \\
\hline Less than 20,000 & 475 & 21.4 & $4.78(2.70)$ & & \\
\hline $20,000-60,000$ & 832 & 37.6 & $4.74(2.67)$ & & \\
\hline $60,000-100,000$ & 516 & 23.3 & $4.90(2.74)$ & & \\
\hline More than 100,000 & 392 & 17.7 & $4.95(2.82)$ & & \\
\hline Infected by COVID-19 & & & & 34.80 & $<0.001^{* *}$ \\
\hline Yes & 54 & 2.4 & $6.96(2.15)$ & & \\
\hline No & 2161 & 96.5 & $4.77(2.71)$ & & \\
\hline $\begin{array}{l}\text { Friends/colleagues/relatives } \\
\text { quarantined due to COVID-19 }\end{array}$ & & & & 15.91 & $<0.001^{* *}$ \\
\hline Yes & 132 & 6.0 & $5.73(2.64)$ & & \\
\hline No & 2083 & 94.0 & $4.76(4.72)$ & & \\
\hline Neighborhood quarantined due to COVID-19 & & & & 24.41 & $<0.001^{* *}$ \\
\hline Yes & 278 & 12.6 & $5.57(2.67)$ & & \\
\hline
\end{tabular}


Table 1 Demographic characteristics and total score of general sleep status (Continued)

\begin{tabular}{llllll}
\hline Variables & $\mathbf{N}$ & \% & Mean (SD) & F/t & p \\
\hline No & 1937 & 87.4 & $4.71(2.71)$ & & \\
\hline${ }^{*}<0.05 ;^{* *}<0.01$ & & &
\end{tabular}

quarantine status due to infection or suspected infection by COVID-19 were all significantly correlated with sleep status.

Table 2 demonstrated the sleep status of participants during the early stage of the COVID-19 outbreak. Among the total sample, $47.1 \%$ went to bed after 12:00 am, $23.0 \%$ took more than $30 \mathrm{~min}$ to fall asleep, and $30.3 \%$ of participants' sleep duration was less than $7 \mathrm{~h}$. A minority of participants (8.5\%) used sleeping medication and $20.9 \%$ rated their sleep quality bad or very bad. Half of the participants experienced daytime dysfunction.

The descriptive statistics and bivariate correlations were displayed in Table 3. The mean total score of sleep status was 4.82 (95\% C.I. 4.71-4.94). Each item score for intolerance of uncertainty, uncertainty about COVID-19, and perceived stress were 3.03 (95\% C.I. 3.00-3.06), 3.08 (95\% C.I. 3.05-3.10), and 2.92 (95\% C.I. 2.90-2.94), respectively. The correlation between intolerance of uncertainty and uncertainty about COVID-19 $(r=0.506, p<$ $0.001)$ and perceived stress and poor sleep $(r=0.336$, $\mathrm{p}<0.001$ ) were positive. Meanwhile, intolerance of uncertainty $(\mathrm{r}=0.538, \mathrm{p}<0.001)$ and uncertainty about COVID-19 $(r=0.360, p<0.001)$ also positively related to perceived stress.

The findings from serial multiple mediation analysis using the bootstrapping method were presented in Tables 4 and 5 . The total effect of COVID-19 uncertainty

Table 2 The sleep status of participants during the early stage of COVID-19 outbreak

\begin{tabular}{|c|c|c|c|c|c|}
\hline Variables & $\mathbf{n}$ & $\%$ & Variables & $\mathrm{n}$ & $\%$ \\
\hline Bedtime & & & \multicolumn{3}{|c|}{ Subjective Sleep quality } \\
\hline Before 11:00 pm & 550 & 24.8 & Very good & 527 & 23.8 \\
\hline 11:00 pm-12:00 am & 622 & 28.1 & Good & 1226 & 55.3 \\
\hline $12: 00 \mathrm{am}-1: 00 \mathrm{am}$ & 589 & 26.6 & Bad & 411 & 18.6 \\
\hline After 1:00 am & 454 & 20.5 & Very Bad & 51 & 2.3 \\
\hline \multicolumn{3}{|l|}{ Sleep latency } & \multicolumn{3}{|c|}{ Use of sleeping medication } \\
\hline$\leq 15$ mins & 855 & 38.6 & None & 2026 & 91.5 \\
\hline $16-30 \mathrm{~min}$ & 851 & 38.4 & $<1 /$ per week & 90 & 4.1 \\
\hline $31-60 \mathrm{~min}$ & 284 & 12.8 & 1-2 /per week & 81 & 3.7 \\
\hline$\geq 60$ mins & 225 & 10.2 & $\geq 3$ / per week & 18 & 0.8 \\
\hline \multicolumn{3}{|l|}{ Sleep duration } & \multicolumn{3}{|c|}{ Daytime dysfunction } \\
\hline$<5 \mathrm{~h}$ & 58 & 2.6 & None & 1108 & 50.0 \\
\hline $5-6 h$ & 187 & 8.4 & $<1 /$ per week & 515 & 23.3 \\
\hline $6-7 \mathrm{~h}$ & 426 & 19.2 & 1-2 /per week & 347 & 15.7 \\
\hline$\geq 7 \mathrm{~h}$ & 1544 & 69.7 & $\geq 3$ / per week & 245 & 11.1 \\
\hline
\end{tabular}

on sleep was significant $(\beta=0.067,95 \%$ C.I. $=0.050$ 0.084) after adjusting potential covariates including demographic characteristics. The results of the mediation analysis confirmed the mediating role of perceived stress in the relationship between COVID-19 uncertainty and general sleep status $(\beta=0.015,95 \%$ C.I. $=0.009$ 0.021 ). However, IU was not a significant mediator of the relationship between COVID-19 uncertainty and sleep $(\beta=0.009,95 \%$ C.I. $=-0.002-0.020)$.

Based on the results of mediation analysis and previous related literature, the path analysis was also conducted to assess the hypothesized network relationships among the variables. With adding the path from uncertainty about COVID-19 to general sleep status, the modified model in Fig. 1 was fitted the data better across the whole samples $(\mathrm{X} 2 / \mathrm{df}=2.377, \mathrm{CFI}=0.999, \mathrm{GFI}=$ 0.999 , RMSEA $=0.025$, SRMR $=0.008$ ). Specifically, uncertainty about COVID-19 had a weak direct effect on poor sleep $(\beta=0.043, p<0.05)$. Uncertainty about COVID-19 had an indirect effect on poor sleep through intolerance of uncertainty $(\beta=0.506, p<0.001)$ and perceived stress $(\beta=0.479, \mathrm{p}<0.001)$. Further, uncertainty about COVID-19 also had another indirect pathway impacting poor sleep via perceived stress $(\beta=0.118, \mathrm{p}<$ 0.001).

\section{Discussion}

The COVID-19 brings unprecedented uncertainty to individuals and society at the initial stage of the outbreak. Uncertainty is an ever-present feature when people face the COVID-19 outbreak. This study investigated the general sleep status of Chinese residents during the third week of lockdown and identified the potential risk factors for influencing sleep from the perspective of uncertainty. We found urban residents, the minority, and the divorced/widowed people had a higher total score of sleep status, suggesting they experienced worse sleep. The government should pay more attention to these populations and their sleep health issues during the COVID-19 pandemic. Participants who were infected by COVID-19 and whose friends, relatives, colleagues, or neighbors were quarantined due to infection or suspected infection by COVID-19 had a significantly higher sleep score than their counterparts. This was in line with the results from other studies showing patients infected with COVID-19 appeared to be the most affected group with a prevalence of sleep problems [5].

Our results from path analysis showed that this kind of uncertainty caused by COVID-19 might have 
Table 3 Inter-correlations and descriptive statistics of study variables

\begin{tabular}{|c|c|c|c|c|c|c|c|c|c|}
\hline & \multicolumn{5}{|l|}{ Variables } & \multicolumn{2}{|l|}{ Total Score } & \multicolumn{2}{|l|}{ Item Score } \\
\hline & & 1 & 2 & 3 & 4 & $M(S D)$ & 95\% C.I. & $M(S D)$ & 95\% C.I. \\
\hline 1 & Intolerance of uncertainty & 1.00 & & & & $36.40(8.19)$ & $36.06-36.74$ & $3.03(0.68)$ & $3.00-3.06$ \\
\hline 2 & Uncertainty about COVID-19 & $0.506^{* *}$ & 1.00 & & & $30.77(6.47)$ & $30.50-31.04$ & $3.08(0.65)$ & $3.05-3.10$ \\
\hline 3 & Perceived stress & $0.538^{* *}$ & $0.360^{* *}$ & 1.00 & & $40.87(6.81)$ & $40.58-41.15$ & $2.92(0.49)$ & $2.90-2.94$ \\
\hline 4 & General sleep status & $0.218^{* *}$ & $0.159^{* *}$ & $0.336^{* *}$ & 1.00 & $4.82(2.72)$ & $4.71-4.94$ & / & / \\
\hline
\end{tabular}

influenced the public's sleeping behaviors and sleep quality. The Illness Uncertainty Theory explains how patients cognitively process an illness-related stimulus, as well as how they structure the meaning of such an event, and proposes that high uncertainty is associated with diminished capacity to process new information, predict outcomes, and adapt to the illness [42]. Not only the illness uncertainty and fear of disease progression affect psychosocial adjustment and the quality of life [43], but the uncertain social situation caused by COVID-19 would also disrupt the people's routine sleep schedule. A recent study revealed that uncertainty about COVID-19 was negatively correlated with general response behaviors including eating and exercise [34]. The possible mechanism was that COVID-19 related uncertainty would cause cognitive confusion, exhaust an individual's energy, diminish perceived control, and divert attention from routine healthy behaviors which might affect regular sleep patterns as well $[34,44]$.

However, we found an effect size smaller than expected for the correlation between uncertainty about COVID-19 and poor sleep status. For possible potential explanation, its key mechanism of the relationship between uncertainty about COVID-19 and poor sleep might be mediated by intolerance of uncertainty and corresponding stress indirectly. The current study revealed higher uncertainty about COVID-19 was significantly positively correlated with higher intolerance of uncertainty, which further increased perceived stress, and subsequently weakened sleep status. COVID-19 is a new virus, related information is unknown or imperfect, and there is much ambiguity surrounding the disease [44]. A host of evidence supports that uncertainty is even more intolerable than knowing the inevitability of something bad happening [44, 45]. Uncertainty about COVID-19 involved both disease uncertainty and unpredictability for returning to normal social life which might results in a perceived fear and intolerance [21]. This high correlation between COVID-19 uncertainty and IU may also be explained by a risk-averse and certainty-oriented culture [34]. Chinese people tend to take uncertainty about COVID-19 as a continuous threat and then become more intolerable with it.

Along with the previous studies, our study has found a positive correlation between IU and perceived stress [19, $21,31,46]$. The link between uncertainty and stress theoretically suggested that IU could increase the negative impact of stressors $[31,47]$. The relationship between IU and psychological distress was also examined among the general population during the first wave of the COVID19 pandemic in the United Kingdom [22]. IU was predictive of mental health difficulties such as depression, anxiety, and fear and this was also mediated by their coping responses [22]. Those mental health difficulties were highly associated with stress and maladaptive coping would, in turn, exacerbate the perceived stress.

In consistent with prior sleep researches, it has been shown that stress uniquely predicts poor sleep quality

Table 4 The results from mediation analysis using a bootstrapping method for general sleep status

\begin{tabular}{|c|c|c|c|c|c|c|c|}
\hline Dependent variable & Independent Variable\# & Beta coefficient & SE & t & $p$ & $\mathrm{R} 2$ & $p$ \\
\hline \multirow[t]{2}{*}{ IU } & Constant & 19.434 & 2.54 & 7.64 & $<0.001^{* *}$ & 0.26 & $<0.001^{* *}$ \\
\hline & Uncertainty about COVID-19 & 0.642 & 0.02 & 27.48 & & & \\
\hline \multirow[t]{3}{*}{ Perceived stress } & Constant & 24.991 & 2.07 & 12.09 & $<0.001^{* *}$ & 0.31 & $<0.001^{* *}$ \\
\hline & IU & 0.397 & 0.02 & 23.25 & $<0.001^{* *}$ & & \\
\hline & Uncertainty about COVID-19 & 0.125 & 0.02 & 5.75 & $<0.001^{* *}$ & & \\
\hline \multirow[t]{4}{*}{ General sleep status } & Constant & 3.723 & 0.95 & 3.92 & $<0.001^{* *}$ & 0.14 & $<0.001^{* *}$ \\
\hline & Perceived stress & 0.119 & 0.01 & 12.57 & $<0.001^{* *}$ & & \\
\hline & IU & 0.014 & 0.01 & 1.61 & 0.11 & & \\
\hline & Uncertainty about COVID-19 & 0.013 & 0.01 & 1.33 & 0.19 & & \\
\hline
\end{tabular}

\# All models were adjusted for infection, quarantine status, and demographic characteristics, such as age, ethnicity, residence, marriage. Bootstrapping = 5000; $* 0.05 ;{ }^{* *}<0.01$ 
Table 5 Indirect effect of uncertainty about COVID-19 on sleep status via intolerance of uncertainty and perceived stress

\begin{tabular}{llll}
\hline Path & Coefficient & \multicolumn{2}{c}{$\mathbf{9 5 \% \text { confidence interval }}$} \\
\cline { 3 - 4 } & & Boot lower limit & Boot upper limit \\
\hline Uncertainty about COVID-2019 $\rightarrow I U \rightarrow$ Sleep & 0.009 & -0.002 & 0.020 \\
Uncertainty about COVID-2019 $\rightarrow$ Perceived stress $\rightarrow$ Sleep & 0.015 & 0.009 & 0.021 \\
Uncertainty about COVID-2019 $\rightarrow I U \rightarrow$ Perceived stress $\rightarrow$ Sleep & 0.030 & 0.024 & 0.037 \\
Total effect & 0.067 & 0.050 & 0.084 \\
Direct effect & 0.013 & -0.006 & 0.032 \\
Total indirect effect & 0.054 & 0.043 & 0.066 \\
\hline
\end{tabular}

regardless of whether it is related to COVID-19 or not $[28,33]$. A Chinese online survey conducted from February 18 to 25,2020 which was close to the date of our investigation found that around one-third of participants were poor sleepers and further indicated perceived stress affected sleep quality through anxiety [33]. Based on Stress and Coping Theory, people perceived stress when taking COVID-19 related events as an environmental stimulus which results in a changed emotional stage such as anxiety that, in turn, affects one's health including sleep quality [33]. Furthermore, previous research has overlooked the role of cognitive dispositions that might exacerbate stress, thus contributing to the continuation of sleep problems [15]. Perceived uncertainty and IU might be such dispositions.

Nevertheless, it is worth noting that IU was not correlated with sleep directly. To our knowledge, IU has been less studied with sleep problems [28] and only limited studies examined IU as antecedents of sleep problems. One study showed IU was strongly associated with anxiety sensitivity, in turn influencing both insomnia severity and sleep quality via depression and anxiety [15]. IU might be involved in the process that links personality to sleep problems as well [15]. The Uncertainty and Anticipation Model of Anxiety identified that individual differences in IU are related to physiological indicators of responses to uncertainty, that are associated with increased risk for anxiety [48, 49]. Another study demonstrated that worry partially mediated the relationship between IU and poor sleep quality in an adolescent sample [28]. In our study, perceived stress mediated the indirect effect of IU on sleep status in a general community sample.

Another pathway of COVID-19 uncertainty influencing sleep in this study was mediated by perceived stress. A panel study showed uncertainty stress was positively associated with disease fear, and negatively associated with self-efficacy, and prevention behaviors, which might lead to irregular sleep status as maladaptive coping strategies [44]. Our study has confirmed perceived stress is a strong factor affecting sleep status. Given the great impact of the individual ability to tolerate uncertainty on the residents' level of perceived stress, it would be worth paying particular attention to such a skill when facing the COVID-19. The practical implications of the present study exhibited that it would be crucial to introduce or to empower tools and strategies that could increase residents' ability to tolerate uncertainty and cope with related stress, further to improve the sleep status. In addition, interventions on the public's training and education to have better access to information about COVID19 and being able to process it adequately may be a possible and alternative strategy for increasing sleep quality. Recently, work by our group has focused on

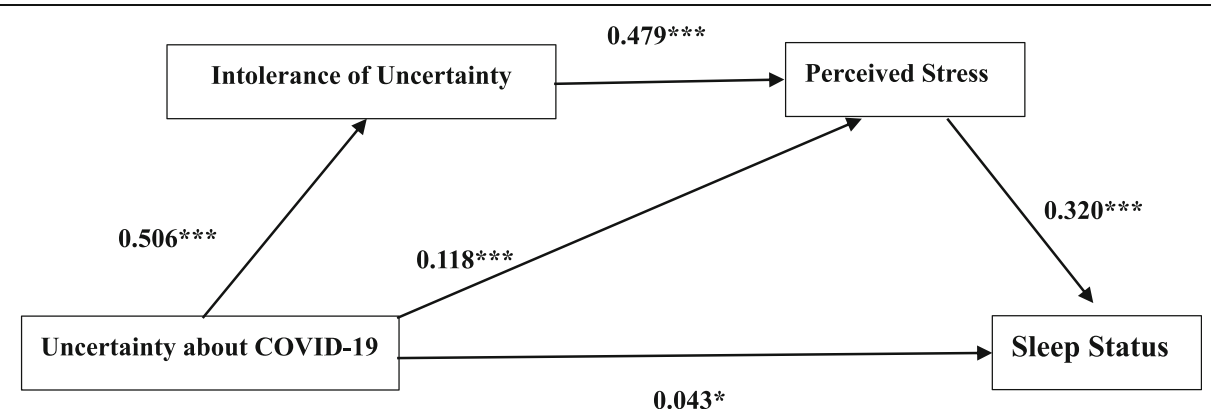

Fig. 1 Path analysis for Uncertainty-Stress-Sleep Model during the early stage of COVID-19 outbreak. ${ }^{*}<0.05 ;{ }^{* *}<0.01$; ${ }^{* *}<0.001$; Values shown are standardized coefficients. $(X 2 / d f=2.377, \mathrm{GFI}=0.999, \mathrm{CFI}=0.999, \mathrm{RMSEA}=0.025, \mathrm{SRMR}=0.008)$ 
disseminating mind-body resiliency skills aimed at reducing intolerance of uncertainty and improving stress management skills using synchronous, virtual platforms that can be accessed while engaging in social distancing [50]. Among cancer survivors, another population facing illness uncertainty, training in relaxation, meditation, cognitive-behavioral coping techniques, and positive psychology skills (e.g., gratitude, using humor) have been associated with favorable improvements in tolerance of uncertainty [51]. Similar resiliency training approaches may thus yield downstream benefits on improving sleep during the COVID-19 pandemic, although to the best of our knowledge this remains unexamined.

\section{Strengths and limitations}

This study has several strengths and limitations. First, general sleep status was assessed via self-report, which may be susceptible to recall bias. Still, the Pittsburgh Sleep Quality Index is psychometrically strong, and objective sleep measures (e.g., actigraphy) have been critiqued for not accounting for perceived sleep disturbances. Second, this report included a large sample of over 2500 Chinese residents. While the crosssectional analyses precluded our ability to draw causal conclusions, our use of structural equation modeling capitalized on the large sample size to test multiple indirect pathways from COVID-19 uncertainty to sleep quality. Third, while we were able to collect surveys during the early stages of the pandemic, we recognized that the over-representation of college students and participants from Guangdong Province suggests sampling bias in this sample which may not generalize to the whole Chinese population, as well as the experiences of uncertainty in other nations whose governments responded differently to managing COVID-19. Finally, the study did not include a measure of mental health problems or specific sleep diseases such as insomnia. Inclusion might have permitted examination of the impact of COVID-19 uncertainty and IU on both overall sleep status and specific sleep-related disorder, after controlling for some other important psychological and behavioral factors.

\section{Conclusions}

The current study suggested that the Chinese residents' uncertainty about COVID-19 is directly associated with poor sleep but also indirectly correlated through the mediating role of IU and perceived stress. This study might provide evidence-based information to the governments and policymakers to design effective health intervention strategies targeted at reducing the COVID-19 uncertainty, improvements in tolerance of uncertainty and coping with related stress, further to improve the sleep status during the stage of COVID-19 pandemic.

\section{Supplementary Information}

The online version contains supplementary material available at https://doi. org/10.1186/s12888-021-03310-2.

\section{Additional file 1.}

\section{Acknowledgements}

The authors would like to thank the research assistants to help us collect data and the residents who participated in this study.

Authors' contributions

DW and TY designed the study, DW, GJ and CJ collected and analysed the data, DW drafted the manuscript, and DH, CJ, LH, and TY revised the manuscript. The authors read and approved the final manuscript.

\section{Funding}

This study was partially supported by Natural Science Foundation of Guangdong Province of China (Grant No. 2018A030307002), Key Research Projects of Colleges and Universities in Guangdong Province

(2018WZXDM015); and Shenzhen Basic Research Grant (2019SHIBS0003).

Availability of data and materials

Data available on request from the corresponding author.

\section{Declarations}

Ethics approval and consent to participate

The study protocol was approved by the Ethics Committee of Shenzhen University, and all methods were carried out in accordance with relevant guidelines and regulations. The written informed consent was obtained from all participants prior to administration of the questionnaire.

Consent for publication

Not applicable.

\section{Competing interests}

The authors declare that they have no conflict of interest with respect to the research, authorship, and/or publication of this article.

\section{Author details}

${ }^{1}$ School of Psychology, Shenzhen University, Shenzhen 518060, Guangdong, China. 'Women's Hospital/Center for Tobacco Control Research, Zhejiang University School of Medicine, Hangzhou 310058, Zhejiang, China. ${ }^{3}$ Massachusetts General Hospital, Harvard Medical School, Boston, MA 02114, USA. ${ }^{4}$ Department of Psychology/Research Center on Quality of Life and Applied Psychology, Guangdong Medical University, Dongguan 523808, Guangdong, China.

Received: 3 February 2021 Accepted: 27 May 2021

Published online: 14 June 2021

\section{References}

1. World Health Organization. Coronavirus disease 2019 (COVID-19) Situation Report. 2020. https://www.who.int/emergencies/diseases/novel-coronavirus2019/situation-reports.

2. Roosa K, Lee $Y$, Luo R, Kirpich A, Rothenberg R, Hyman JM, et al. Real-time forecasts of the COVID-19 epidemic in China from February 5th to February 24th, 2020. Infect Dis Model. 2020;5:256-63. https://doi.org/10.1016/j.idm.2 020.02 .002 .

3. Gupta R, Grover S, Basu A, Krishnan V, Tripathi A, Subramanyam A, et al. Changes in sleep pattern and sleep quality during COVID-19 lockdown. Indian J Psychiatry. 2020;62(4):370-5. https://doi.org/10.1111/jsr.13074.

4. Gualano MR, Moro GL, Voglino G, Bert F, Siliquini R. Effects of Covid-19 lockdown on mental health and sleep disturbances in Italy. Int J Environ Res Public Health. 2020;17(13):4779. https://doi.org/10.3390/ijerph17134779.

5. Jahrami H, BaHammam AS, Bragazzi NL, Saif Z, Faris M, Vitiello MV. Sleep problems during the COVID-19 pandemic by population: a systematic review and meta-analysis. J Clin Sleep Med. 2021;17(2):299-313. https://doi. org/10.5664/jcsm.8930. 
6. Fu W, Wang C, Zou L, Guo Y, Lu Z, Yan S, et al. Psychological health, sleep quality, and coping styles to stress facing the COVID-19 in Wuhan, China. Transl Psychiatr. 2020;10(1):1-9. https://doi.org/10.1038/s41398-020-00913-3.

7. Voulgaris A, Ferini-Strambi L, Steiropoulos P. Sleep medicine and COVID-19. Has a new era begun? Sleep Med. 2020;73:170-6. https://doi.org/10.1016/j. sleep.2020.07.010.

8. Gao C, Scullin MK. Sleep health early in the coronavirus disease 2019 (COVID-19) outbreak in the United States: integrating longitudinal, crosssectional, and retrospective recall data. Sleep Med. 2020;73:1-10. https://doi. org/10.1016/j.sleep.2020.06.032.

9. lannello P, Mottini A, Tirelli S, Riva S, Antonietti A, et al. Med. Educ. Online. 2017;22(1). https://doi.org/10.1080/10872981.2016.1270009.

10. Greco V, Roger D. Uncertainty, stress, and health. Pers Individ Differ. 2003; 34(6):1057-68. https://doi.org/10.1016/s0191-8869(02)00091-0.

11. Karacan I, Thornby JI, Williams RL. Sleep disturbance: a community survey. In: Guilleminault C, Lugaresi E, editors. Sleep/wake disorders: natural history, epidemiology, and long-term evolution. Raven Press: New York; 1983. p. $37-60$

12. Mishel M. The measurement of uncertainty in illness. Nurs Res. 1981;5(30): 258-63. https://doi.org/10.1097/00006199-198109000-00002.

13. Koocher GP. Psychosocial care of the child cured of cancer. J Spec Pediatr Nurs. 1985;11:91-3.

14. Buhr KK, Dugas MJ. Investigating the construct validity of intolerance of uncertainty and its unique relationship with worry. J Anxiety Disord. 2006; 20:222-36. https://doi.org/10.1016/j.janxdis.2004.12.004.

15. Lauriola M, Carleton RN, Tempesta D, Calanna P, Socci V, Mosca O, et al. A correlational analysis of the relationships among intolerance of uncertainty, anxiety sensitivity, subjective sleep quality, and insomnia symptoms. Int J Environ Res Public Health. 2019;16(18):3253. https://doi.org/10.3390/ijerph1 6183253.

16. Freeston M, Rhéaume J, Letarte H, Dugas M, Ladouceur R. Why do people worry? Pers Individ Differ. 1994;17(6):791-802. https://doi.org/10.1016/01918869(94)90048-5.

17. Carleton RN. The intolerance of uncertainty construct in the context of anxiety disorders: theoretical and practical perspectives. Expert Rev Neurother. 2012;12(8):937-47. https://doi.org/10.1586/ern.12.82.

18. Carleton RN. Into the unknown: a review and synthesis of contemporary models involving uncertainty. J Anxiety Disord. 2016;39:30-43. https://doi. org/10.1016/j.janxdis.2016.02.007.

19. Zlomke KR, Jeter KM. Stress and worry: examining intolerance of uncertainty's moderating effect. Anxiety Stress Coping. 2014;27(2):202-15. https://doi.org/10.1080/10615806.2013.835400.

20. Wu D, Yu L, Yang T, Cottrell R, Peng S, Guo W, et al. The impacts of uncertainty stress on mental disorders of Chinese college students: evidence from a nationwide study. Front Psychol. 2020;11(243):1-9. https:// doi.org/10.3389/fpsyg.2020.00243.

21. Bakioğlu F, Korkmaz O, Ercan H. Fear of COVID-19 and positivity: mediating role of intolerance of uncertainty, depression, anxiety, and stress. Int J Mental Health Addict. 2020:1-14. https://doi.org/10.1007/s11469-02000331-y.

22. Rettie H, Daniels J. Coping and tolerance of uncertainty: predictors and mediators of mental health during the COVID-19 pandemic. Am Psychol. 2020:1-12. https://doi.org/10.1037/amp0000710.

23. Deniz ME. Self-compassion, intolerance of uncertainty, fear of COVID-19, and well-being: a serial mediation investigation. Pers Individ Differ. 2021;177: 110824. https://doi.org/10.1016/j.paid.2021.110824.

24. Behar E, DiMarco ID, Hekler EB, Mohlman J, Staples AM. Review: current theoretical models of generalized anxiety disorder (GAD): conceptual review and treatment implications. J Anxiety Disord. 2009;23(8):1011-23. https://doi. org/10.1016/j.janxdis.2009.07.006

25. McEvoy PM, Hyett MP, Shihata S, Price JE, Strachan L. The impact of methodological and measurement factors on transdiagnostic associations with intolerance of uncertainty: a meta-analysis. Clin Psychol Rev. 2019;73: 101778. https://doi.org/10.1016/j.cpr.2019.101778.

26. Sabouri S, Gerber M, Lemola S, Becker SP, Shamsi M, Shakouri Z, et al. Examining dark triad traits in relation to sleep disturbances, anxiety sensitivity and intolerance of uncertainty in young adults. Compr Psychiatry. 2016;68:103-10. https://doi.org/10.1016/j.comppsych.2016.03.012.

27. Tsypes A, Aldao A, Mennin DS. Emotion dysregulation and sleep difficulties in generalized anxiety disorder. J Anxiety Disord. 2013;27(2):197-203. https:// doi.org/10.1016/j.janxdis.2013.01.008.
28. Lin RM, Xie SS, Yan YW, Yan WJ. Intolerance of uncertainty and adolescent sleep quality: the mediating role of worry. Pers Individ Differ. 2017;108:16873. https://doi.org/10.1016/j.paid.2016.12.025.

29. Hall DL, Mishel MH, Germino BB. Living with cancer-related uncertainty: associations with fatigue, insomnia, and affect in younger breast cancer survivors. Support Care Cancer. 2014;22(9):2489-95. https://doi.org/10.1007/ s00520-014-2243-y.

30. Dugas MJ, Gosselin P, Ladouceur R. Intolerance of uncertainty and worry: investigating specificity in a nonclinical sample. Cognitive Ther Res. 2001; 25(5):551-8. https://doi.org/10.1023/A:1005553414688.

31. Demirtas AS, Yildiz B. Hopelessness and perceived stress: the mediating role of cognitive flexibility and intolerance of uncertainty. Dusunen Adam The Journal of Psychiatry and Neurological Sciences. 2019;32:259-67. https://doi. org/10.14744/DAJPNS.2019.00035.

32. Åkerstedt T, Orsini N, Petersen H, Axelsson J, Lekander M, Kecklund G. Predicting sleep quality from stress and prior sleep - a study of day-to-day covariation across six weeks. Sleep Med. 2012;13(6):674-9. https://doi.org/1 0.1016/j.sleep.2011.12.013.

33. Zhao X, Lan M, Li H, Yang J. Perceived stress and sleep quality among the non-diseased general public in China during the 2019 coronavirus disease: a moderated mediation model. Sleep Med. 2021;77:339-45. https://doi.org/1 0.1016/j.sleep.2020.05.021.

34. Wu D, Rockett IR, Yang T, Yang XY, Wang M, Jiao C. Perceived beliefs, uncertainty, and behavioral responses during the COVID-19 outbreak in China: findings from a convenience sample. Am J Health Promotion. 2021: 08901171211004249. https://doi.org/10.1177/08901171211004249.

35. Buysse DJ, lii CFR, Monk TH, Berman SR, Kupfer DJ. The Pittsburgh sleep quality index: a new instrument for psychiatric practice and research. Psychiatry Res. 1989;28(2):193-213. https://doi.org/10.1016/0165-1781 (89)90047-4.

36. Carleton RN, Norton MAPJ, Asmundson GJG. Fearing the unknown: a short version of the intolerance of uncertainty scale. J. Anxiety Disord. 2007;21(1): 105-17. https://doi.org/10.1016/j.janxdis.2006.03.014.

37. Hale W, Richmond M, Bennett J, Berzins T, Fields A, Weber D, et al. Resolving uncertainty about the intolerance of uncertainty scale-12: application of modern psychometric strategies. J Pers Assess. 2016;98(2): 200-8. https://doi.org/10.1080/00223891.2015.1070355.

38. Cohen $\mathrm{S}$, Kamarack T, Mermelstein R. A global measure of perceived stress. J Health Soc Behav. 1983;24(4):385-96. https://doi.org/10.2307/2136404.

39. Yang T, Wu D, Zhang WF, Cottrell RR, Rockett IRH. Comparative stress levels among residents in three Chinese provincial capitals, 2001 and 2008. PLoS One. 2012;7(11):5225-35. https://doi.org/10.1371/journal.pone.0048971.

40. Ullman JB, Bentler PM. Structural equation modeling. Handb Psychol. 2003: 607-34. https://doi.org/10.1002/0471264385.wei0224.

41. Weston R, Gore PA Jr. A brief guide to structural equation modeling. Couns Psychol. 2006;34(5):719-51. https://doi.org/10.1177/0011000006286345.

42. Mishel MH. Reconceptualization of the uncertainty in illness theory. Image J Nurs Scholarship. 1990;22(4):256-62. https://doi.org/10.1111/j.1547-5069.1 990.tb00225.x.

43. Sharif SP. Locus of control, quality of life, anxiety, and depression among Malaysian breast cancer patients: the mediating role of uncertainty. Eur J Oncol Nurs. 2017;27:28-35. https://doi.org/10.1016/j.ejon.2017.01.005.

44. Peng S, Yang XY, Yang T, Zhang W, Cottrell RR. Uncertainty stress, and its impact on disease fear and prevention behavior during the COVID-19 epidemic in China: a panel study. Am J Health Behav. 2021;45(2):334-41. https://doi.org/10.5993/AJHB.45.2.12.

45. Yang T. Heath research: social and behavioral theory and methods. Beijing: People's Medical Publishing House; 2018.

46. Lally J, Cantillon P. Uncertainty and ambiguity and their association with psychological distress in medical students. Acad Psych. 2014;38(3):339-44. https://doi.org/10.1007/s40596-014-0100-4.

47. Chen CY, Hong RY. Intolerance of uncertainty moderates the relation between negative life events and anxiety. Pers Individ Differ. 2010;49:49-53. https://doi.org/10.1016/j.paid.2010.03.006

48. Grupe DW, Nitschke JB. Uncertainty and anticipation in anxiety: an integrated neurobiological and psychological perspective. Nat Rev Neurosci. 2013;14(7):488-501. https://doi.org/10.1038/nrn3524.

49. Tanovic E, Gee DG, Joormann J. Intolerance of uncertainty: neural and psychophysiological correlates of the perception of uncertainty as threatening. Clin Psychol Rev. 2018;60:87-99. https://doi.org/10.1016/j.cpr.2 018.01.001. 
50. Hall DL, Millstein RA, Luberto CM, Perez GK, Park ER. Responding to COVID19 stress: disseminating mind-body resiliency approaches. Glob Adv Health Med. 2020;9:1-4. https://doi.org/10.1177/2164956120976554.

51. Hall DL, Park ER, Cheung T, Davis RB, Yeh GY. A pilot mind-body resiliency intervention targeting fear of recurrence among Cancer survivors. J Psychosom Res. 2020;137:110215. https://doi.org/10.1016/j.jpsychores.202 0.110215 .

\section{Publisher's Note}

Springer Nature remains neutral with regard to jurisdictional claims in published maps and institutional affiliations.

Ready to submit your research? Choose BMC and benefit from:

- fast, convenient online submission

- thorough peer review by experienced researchers in your field

- rapid publication on acceptance

- support for research data, including large and complex data types

- gold Open Access which fosters wider collaboration and increased citations

- maximum visibility for your research: over $100 \mathrm{M}$ website views per year

At $\mathrm{BMC}$, research is always in progress.

Learn more biomedcentral.com/submissions 